\title{
Quasars, supermassive black holes, and host galaxy evolution
}

\author{
Fred Hamann ${ }^{1}$, Craig Warner ${ }^{1}$ \\ and Matthias Dietrich ${ }^{2}$ \\ ${ }^{1}$ Department of Astronomy, University of Florida, Gainesville, FL 32611-2055, USA \\ ${ }^{2}$ Department of Physics and Astronomy, Georgia State University, 29 Peachtree Center \\ Avenue, Science Annex, Suite 400, Atlanta, GA 30303-4106, USA
}

\begin{abstract}
We discuss some recent results from a program to study the relationships between AGNs, super-massive black holes (SMBHs), and their surrounding host galaxies. The results are based on broad emission-line diagnostics of the SMBH masses and metal abundances in a unique sample of 578 Type 1 AGNs.
\end{abstract}

\section{Introduction}

High-redshift quasars are bright, short-lived beacons that signify the formation (or rapid growth) of super-massive black holes (SMBHs) in the cores of young massive galaxies. Measurements of the gas-phase abundances near quasars can probe the evolutionary relationships between galaxies, quasars, and SMBHs by placing constraints on the maturity of the surrounding galactic environments. For example, the overall metallicity depends on the extent of prior star formation, e.g., on the fraction of the initial (primordial) gas that has already been converted to stars and stellar remnants at the quasar epoch (modulo uncertainties in the stellar initial mass function and the infall or outflow of gas from the region, Hamann et al. 2004, Hamann \& Ferland 1999). Quasar abundance studies can therefore address fundamental questions, such as, when did the first major star formation begin in massive galactic nuclei, e.g., with respect to the epochs of SMBH growth and visible quasar/AGN activity? This question is of particular interest because the first major star formation associated with high-redshift quasars is likely to be the first major star formation anywhere in the universe. Galactic (or proto-galactic) nuclei that are massive and dense enough to produce SMBHs with masses $M_{B H}>10^{8} \mathrm{M}_{\odot}$ at high redshifts are the most likely sites of major starbursts soon after the Big Bang.

We can also examine the relationships of AGN metallicities and emission-line properties to other AGN parameters, such as $M_{B H}$, luminosity $L$, and accretion efficiency $L / L_{e d d}$, where $L_{e d d}$ is the Eddington luminosity assuming spherical symmetry. For example, it has been known for some time that the broad emission-line ratios used to infer metallicity, such as NV $\lambda 1240 / \mathrm{CIV} \lambda 1549$, change in a way that suggests higher metallicities at higher AGN luminosities (Hamann \& Ferland 1999). In Warner et al. (2003) we showed that these same line ratios also increase with SMBH mass, and we speculated that galaxy mass, not AGN luminosity, is the fundamental driver behind AGN metallicities. This speculation is based on the well-established relationships between galaxy mass and metallicity (Trager et al. 2000), and between galaxy mass and $M_{B H}$ of the central SMBH (Gebhardt et al. 2000). If more massive galaxies produce higher metallicities and more massive SMBHs, then their AGNs should be more metal rich - as long as the enrichment precedes the visible AGN phase. Here we present some critical tests of these ideas. 


\section{Sample Definition and Results}

The data sample includes 578 type I (broad-line) AGNs for which we have spectra covering the rest wavelengths $950<\lambda<2050 \AA$. The spectra were obtained from the Hubble Space Telescope archives and from a variety of ground-based observatories (see Dietrich et al. 2002 and Warner et al. 2003 for details). The AGNs span 5 orders of magnitude in luminosity and the redshift range $0<z<5$. One unique aspect of this sample is that it includes faint, low-luminosity AGNs at high redshifts, so the effects of redshift and luminosity can be studied independently.

We derive SMBH masses for every object in the sample by assuming the velocities in the broad emission line region (BELR) are virialized and the radial distance of the BELR from the SMBH scales approximately as $R_{B E L R} \propto L^{0.7}$ (Kaspi et al. 2000). The full width at half maximum (FWHM) of CIV $\lambda 1549$ defines the BELR velocities, leading to

$$
M_{B H}=1.4 \times 10^{6}\left(\frac{\mathrm{FWHM}(\mathrm{CIV})}{1000 \mathrm{~km} / \mathrm{s}}\right)^{2}\left(\frac{\lambda L_{\lambda}(1450 \AA)}{10^{44} \mathrm{ergs} / \mathrm{s}}\right)^{0.7} \mathrm{M}_{\odot}
$$

The accretion efficiency is then given by

$$
\frac{L}{L_{e d d}}=1.6\left(\frac{\mathrm{FWHM}(\mathrm{CIV})}{1000 \mathrm{~km} / \mathrm{s}}\right)^{-2}\left(\frac{\lambda L_{\lambda}(1450 \AA)}{10^{44} \mathrm{ergs} / \mathrm{s}}\right)^{0.3}
$$

To isolate the effects of mass and luminosity, we first consider a sample of AGNs in the narrow luminosity range, $3 \times 10^{46} \leqslant L \leqslant 3 \times 10^{47} \mathrm{~L}_{\odot}$, and create composite spectra in different intervals of SMBH mass. The resulting four composites are plotted in the left panel of Figure 1, with the average SMBH mass for each composite shown along the right edge of the panel. These four composites have the same average luminosity to within a factor of 2.7 , but they span a factor of 18 in average SMBH mass. Notice the strong trends in both the CIV peak height relative to the continuum and the NV/CIV line ratio (i.e., metallicity) with changing $M_{B H}$.

Next we consider AGNs in the narrow SMBH mass range, $8 \times 10^{8} \leqslant M_{B H} \leqslant 2 \times 10^{9} \mathrm{M}_{\odot}$, and create composites at different luminosities. The resulting three composite spectra are shown in the right panel of Figure 1, with the average luminosity in each composite indicated along the right edge. Here composites have the same average SMBH mass to within $26 \%$, but they span a factor of 8 in average $L$. Notice the very different emission line behaviors compared to the left-hand panel in Figure 1, namely, constant line peak heights and constant NV/CIV line ratios (metallicities) with changing $L$.

In Warner et al. (2004b) we examined trends in the emission line properties with $L / L_{e d d}$ by creating composite spectra in different $L / L_{e d d}$ ranges using the entire sample of 578 AGNs. Those results are shown in Figure 2. This figure includes a composite spectrum of 26 narrow-line Seyfert 1s (NLS1s) classified as such by others based on their narrow $\mathrm{H} \beta$ profiles and other properties. Notice the nearly constant line peak heights and constant NV/CIV line ratios (metallicities) among these $L / L_{e d d}$ composites.

Although less obvious from these figures, other metallicity diagnostics, such as NV/HeII $\lambda 1640$ and NIII] $\lambda 1750 / \mathrm{OIII}] \lambda 1663$, also indicate a strong trend for increasing metallicity with SMBH mass, but there are no trends in these line ratios with $L$ or $L / L_{\text {edd }}$ (see Warner et al. 2004a and 2004b).

Finally, a note about the uncertainties in Equations 2.1 and 2.2. One could argue that the $R_{B E L R} \propto L^{0.7}$ scaling is not exact (e.g., Netzer 2003), or that $\operatorname{FWHM}(\mathrm{H} \beta$ ) is more extensively calibrated for $M_{B H}$ estimates than FWHM(CIV) (Peterson \& Wandel 2000). However, the exact form of the $R_{B E L R}-L$ relationship is not important for the main results in Figures 1 and 2. Also, we have shown (Warner et al. 2003) that, in spite 

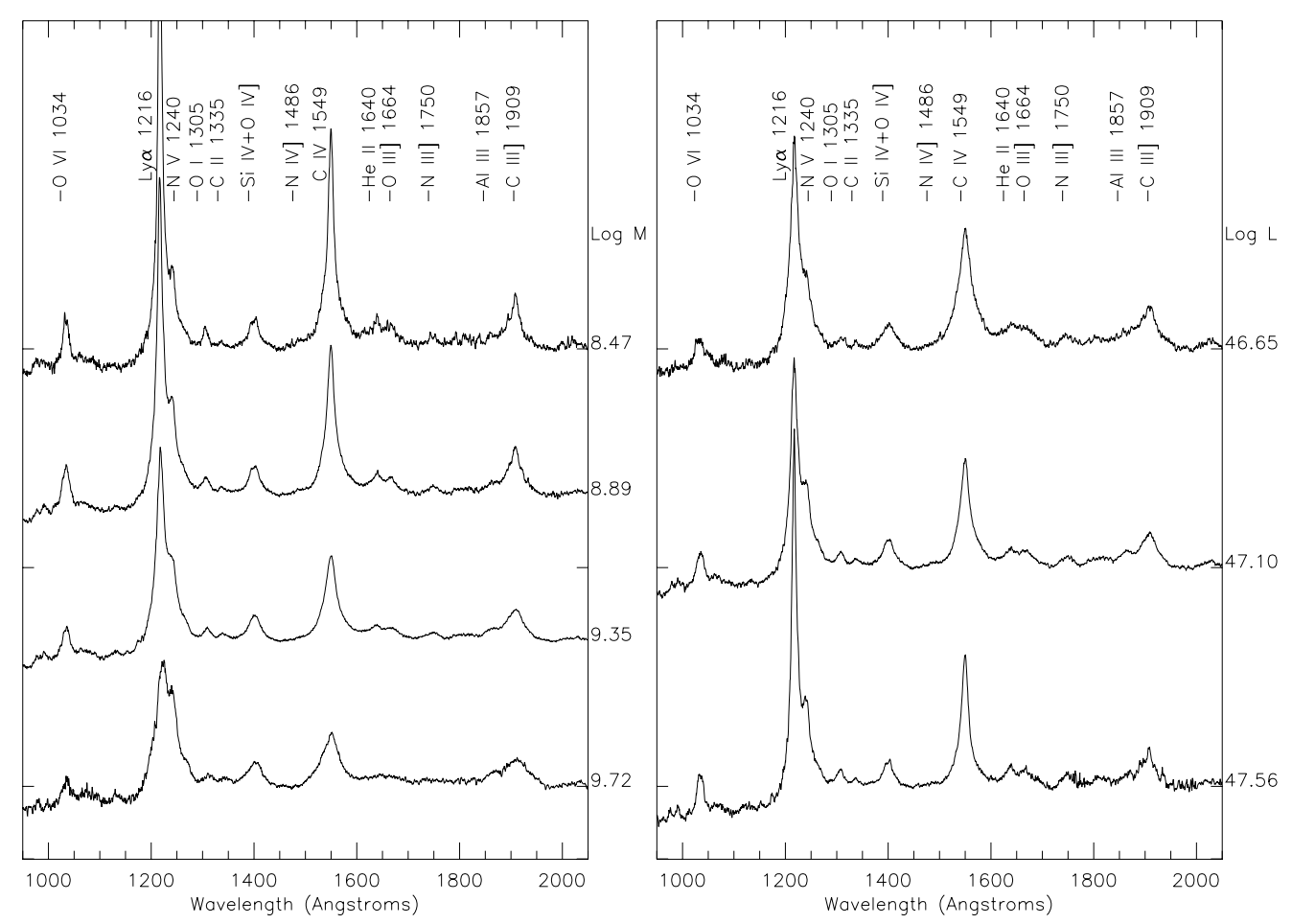

Figure 1. Composite spectra spanning a range of SMBH masses at roughly the same $L \approx 10^{47}$ ergs s${ }^{-1}$ (left panel), and a range of luminosities at fixed $M_{B H} \approx 10^{9} \mathrm{M}_{\odot}$ (right panel). The mean values of $M_{B H}$ and $L$, respectively, corresponding to each spectrum are shown at the right of each panel. The continuum heights above zero flux are the same for every spectrum, but vertical shifts were added for clarity. From Warner et al. (2004a).

of the fairly poor correlation between $\operatorname{FWHM}(\mathrm{CIV})$ and $\operatorname{FWHM}(\mathrm{H} \beta)$, these two lines yield roughly the same values of $M_{B H}$ on average (see also Vestergaard 2002). Even if one took the extreme position that the quantities we call $M_{B H}$ and $L / L_{\text {edd }}$ are nothing more than different parameterizations of the same two observables, $L$ and FWHM(CIV) in Equations 2.1 and 2.2, the different trends that emerge from these parameterizations (Figs. 1 and 2) are real and dramatic and still require explanation.

\section{General Summary}

- Luminous quasars, with $M_{B H} \geqslant$ a few $\times 10^{8} \mathrm{M}_{\odot}$, have metallicities near or above solar even at the highest redshifts, requiring substantial star formation before the bright AGN phase - probably contemporaneous with SMBH growth (Dietrich et al. 2003, 2004).

- The stellar populations/starbursts that rapidly enrich quasar environments at high redshifts probably have masses $>M_{B H}$, corresponding to a significant (central) component of the host galaxies (Baldwin et al. 2003, Hamann et al. 2004).

- The nitrogen line ratios indicative of metallicity correlate strongly with $M_{B H}$, but not with $L$ or $L / L_{e d d}$ (Warner et al. 2004a and b). Host galaxy mass, e.g., of the spheroidal component, is probably the most important factor in determining quasar metallicities.

- NLS1s are slightly more metal rich than other type 1 AGNs of similar $L$ or $M_{B H}$, but this deviation is not driven by differences in $L / L_{e d d}$ (Warner et al. 2004b). 


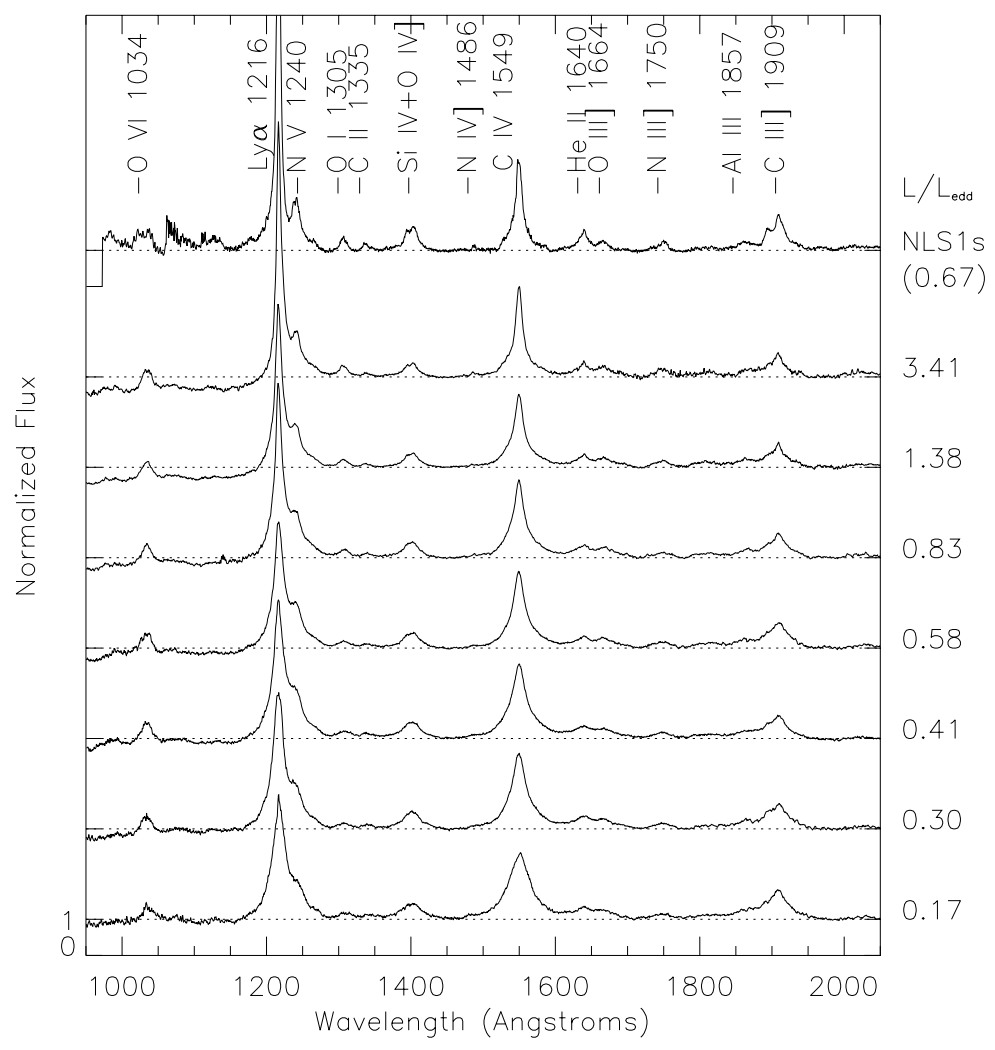

Figure 2. Normalized composite spectra spanning a range in $L / L_{e d d}$, as indicated at the right of each spectrum. The top spectrum is the NLS1 composite, with its mean value of $L / L_{e d d}$ shown in parentheses. The continuum heights above zero flux are the same for every spectrum. From Warner et al. (2004b).

\section{Acknowledgements}

This work was supported by grants from the NSF (AST99-84040) and the Space Telescope Science Institute (AR-07988.01-96A)

\section{References}

Baldwin, J. A., et al. 2003, ApJ, 582, 590

Dietrich, M., \& Hamann, F. 2004, ApJ, submitted

Dietrich, M., et al. 2002, ApJ, 581, 912

Dietrich, M., et al. 2003, ApJ, 589, 722

Gebhardt, K., et al. 2000, ApJ, 539, L13

Hamann, F., \& Ferland, G. J. 1999, ARA\&A, 37, 487

Hamann, F., et al. 2004, Carnegie Observatories Astrophys. Ser., Vol. 4, in press

Kaspi, S., et al. 2000, ApJ, 533, 631

Netzer, H. 2003, ApJ, 583, L5

Peterson, B. M., \& Wandel, A. 2000, ApJ, 540, L13

Trager, S. C., et al. 2000, AJ, 120, 165

Vestergaard, M. 2002, ApJ, 57, 733

Warner, C., Hamann, F., \& Dietrich, M. 2003, ApJ, 596, 72

Warner, C., Hamann, F., \& Dietrich, M. 2004a, in preparation

Warner, C., Hamann, F., \& Dietrich, M. 2004b, ApJ, in press 